\section{Explicit Event}

Jonas Mellin $^{1,2}$ and Mikael Berndtsson ${ }^{1,2}$

${ }^{1}$ University of Skövde, The Informatics

Research Centre, Skövde, Sweden

${ }^{2}$ University of Skövde, School of Informatics,

Skövde, Sweden

\section{Definition}

In active databases, an explicit event is explicitly specified in the ECA rule definition.

\section{Key Points}

ECA rules were developed as an optimization of condition action rules. The performance of rule evaluation was improvedby allowing, or even requiring, explicit definition of when rules should be triggered in the form of events. For example, (ON update $_{A}$ followed_by update ${ }_{B}$ IF $A=5 \wedge$ $B=3$ DO action) is a rule specification that is triggered by the explicit event update ${ }_{A}$ followed_by update ${ }_{B}$ (i.e., a sequence of updates).

\section{Cross-References}

- Atomic Event

- Composite Event

$\checkmark$ ECA Rules

$>$ Event

- Event Detection

$\checkmark$ Event Specification

$>$ Implicit Event 